\title{
Contact geometry during indentation of a sphere into an elastoplastic half-space
}

\author{
Peter Ogar ${ }^{1, *}$, Denis Gorokhov $^{1}$, Artem Zhuk $^{1}$, and Vladislav Kushnarev ${ }^{1}$ \\ ${ }^{1}$ Bratsk State University, Makarenko 40, 665709 Bratsk, Russia
}

\begin{abstract}
The indentation of a sphere into an elastoplastic half-space is considered, which is accompanied by pile-up / sink-in effects, extrusion of material around a sphere (formation of a bulk) and elastic sinking of the material. The evolution of studies of the indicated phenomena is shown. Based on the similarity of the deformation characteristics, expressions are obtained for determining the indentation depth, the depth of the residual crater, and the contact depth depending on the degree of loading. In this case, the influence of the characteristics of the hardened material - the yield strength and the hardening exponent - were taken into account. The radial boundary of the bulk is determined from the volume of the displaced material. Expressions are obtained for describing the profile of a loaded and unloaded crater.
\end{abstract}

\section{Introduction}

The operational performance of the joints of machine parts, including tightness, are determined of the relative contact area and the density of the gaps at the junction of rough surfaces $[1,2]$. For determining contact characteristics at the junction of rough surfaces, various roughness models are widely used, in which microasperities (hereinafter, asperities) are represented as spherical segments with the same radius. Moreover, the asperities height distribution corresponds to the normal law [3] or the surface bearing curve [1, 4]. It should be noted that in [2] the equation of the entire profile bearing curve is used, and not only its initial part as in $[1,2,4]$. For elastic contact of asperities, Hertz theory is used to determine contact characteristics. When the contact characteristics reach critical values, the stress state will begin to cause fluidity inside the body. In most cases, the contact of metal surfaces is elastoplastic. As indicated by the authors of [5], in the past many analytical, experimental, and numerical studies have been performed for modeling and predicting the properties of an elastoplastic contact, such as the contact radius, the average pressure, and the contact strength. However, because of their complexity, no closed solution was proposed for elastoplastic contacts. An exception is the work [6], which was not considered in [5]. From a review of numerical studies [5], it follows that for simplify the problem, most authors model the contact of a sphere and a half-space. Moreover, contact models can be divided into two main groups: indentation models and flattening models [7]. In flattening models, a

\footnotetext{
* Corresponding author: ogar@brstu.ru
} 
plane is considered rigid and the sphere is deformed, while in indentation models, the plane is deformed, and the hemisphere is either rigid or elastic.

The presented results of numerical studies for a separate spherical asperity [5, 8] relate the relative contact area and the relative applied force to the relative displacement in the contact. Given the known asperities height distribution, it is possible to determine the relative contact area of the junction of rough surfaces. Difficulties arise in determining the density (or volume) of gaps in the joint. The volume of gaps in the joint is determined by the total volume of gaps per one asperity, which is determined by the geometry of the contact - the indentation depth of the asperity $h$, the contact depth $h_{c}$ and the profile of the deformable surface. If the problem of determining the density of gaps is solved for an elastic contact of asperities, including under the mutual influence of asperities $[2,9,10]$, then for an elastic-plastic contact this solution is associated with "sink-in / pile-up" effects (Fig. 1). The aim of this work is to determine the contact geometry when introducing a rigid spherical asperity into an elastoplastic hardened half-space.

\section{2 «Sink-in/pile-up» effects}

Mayer was the first who described the behavior of the material in the elastoplastic region, which related the load $P$ to the imprint diameter $d=2 a$ by a power law [11], which is often represented as:

$$
\frac{P}{\pi a^{2}}=A^{*}\left(\frac{a}{R}\right)^{m-2},
$$

where $m$ is the Mayer index, $A^{*}$ is a constant that has a stress dimension.

The expression on the left is the average pressure at the contact area, which is called Mayer hardness.

$$
\frac{P}{\pi a^{2}}=p_{m}=H M
$$

For the first time, attention was paid to the effects of "pile-up / sink-in" (Fig. 1) when measuring hardness according to Brinell Norbury and Samuel [12]. The ratio of $s / h$ where $s=h_{c}-h$, they are associated with the ability of materials to harden.

Given that for a wide range of materials, the true stress under uniaxial deformation is described by a power law

$$
S=k \varepsilon^{n},
$$

where $k$ is the coefficient, $\varepsilon$ is the plastic deformation, $n$ is the exponent of hardening, Matthews [13] proposed the following expression for $a / R=0,4 \ldots 0,8$ :

$$
\frac{s}{h}=\frac{1}{2}\left(1+\frac{n}{2}\right)^{2\left(\frac{1-n}{n}\right)} .
$$

Theoretically studying Brinell hardness using finite element analysis, Hill et al. [14] proposed a new parameter 


$$
c^{2}=\frac{h_{c}}{h}=\frac{5}{2}\left(\frac{2-n}{n+4}\right),
$$

including also only the exponent of hardening.

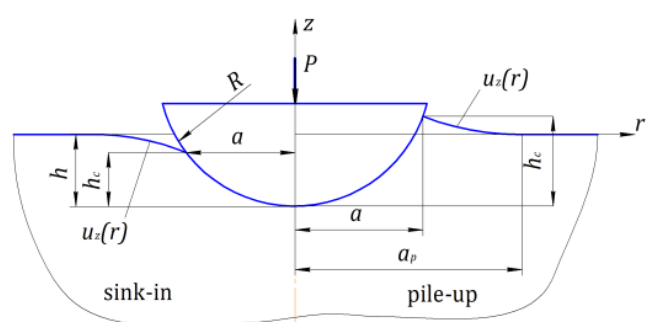

Fig. 1. Pile-up / sink-in effects diagram

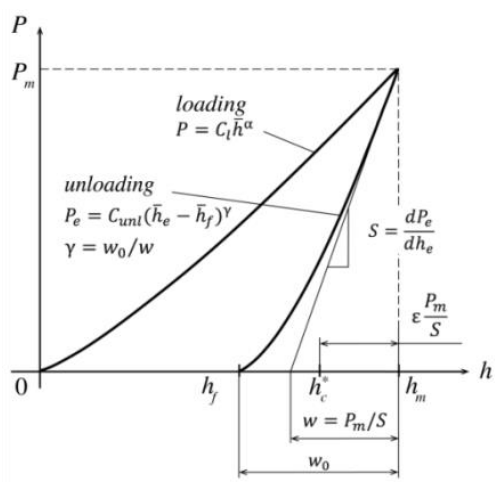

Fig. 2. The kinetic indentation diagram

Further development of the study of the effects of "pile-up / sink-in" was associated with the improvement of the method of measuring hardness and elastic modulus, developed in 1992 by Oliver and Farr $[15,16]$ and adapted for characterization at the micro- and nanoscale. The method is based on the expression for contact stiffness obtained by Bulychev et al., who first proposed the kinetic indentation of materials (Fig. 2) in order to determine their mechanical properties.

According to [17], the contact stiffness of the initial part of the unloading curve

$$
S=\frac{d P_{e}}{d h_{e}}=\frac{2}{\sqrt{\pi}} E^{*} \sqrt{A},
$$

where $A$ is the contact projection area, $E^{*}$ is the contact modulus of elasticity:

$$
E^{*}=\left(\frac{1-v_{i}^{2}}{E_{i}}+\frac{1-v^{2}}{E}\right)^{-1},
$$

$v_{i}, E_{i}$ and $v, E$ are the Poisson's ratio and elastic modulus of the indenter and material, respectively.

In the proposed method [15], the hardness and elastic modulus were determined by the expressions:

$$
H=\frac{P}{A} ; \quad E^{*}=\frac{\sqrt{\pi}}{2 \beta} \cdot \frac{S}{\sqrt{A}},
$$

where $\beta \approx 1,05$ is the correction factor.

As follows from expressions (13), special attention should be paid to the accuracy of determining the projection area of the print, since neglect of the pile-up effect leads to underestimation of the contact area to $60 \%$ [16]. 
Further refinement of the parameter $c^{2}$ occurred for any specific conditions [18, 19, 20]. In the indicated papers, the parameter $c^{2}$ depends only on $n$, and the influence of the material properties $\sigma_{y}$ and $E$ on $c^{2}$ is not shown.

In a later paper [21], Taljat and Pharr focused on a more detailed study of the pile-up effect. Using finite element modeling, they studied the effect of elastic deformation depending on the $E / \sigma_{y}$ ratio, the influence of the relative penetration depth $\bar{h}=h / R$, the exponents of hardening, and the friction coefficient, but they did not present the results in a form convenient for engineering calculations.

In this regard, the papers $[22,23]$ differ in which $c^{2}$ is represented in the form $c^{2}=c^{2}\left(\varepsilon_{y}, n, \bar{h}\right)$, where $\varepsilon_{y}=\sigma_{y} / E, \bar{h}=h / R$. In [22] Lee et al. describe the characteristics of an elastoplastic contact by polynomial functions obtained as a result of finite element analysis. Hernot et al. [23] obtained for spherical indentation:

$$
c^{2}=\frac{h_{c}}{h}=M^{2 / N}(2 \bar{h})^{(2-N) / N},
$$

where $M=M\left(\varepsilon_{y}, n\right), N=N\left(\varepsilon_{y}, n\right)$.

Kim et al. [24] indicated that the contact depth $h_{c}$ can be represented by the addition of two independent terms

$$
h_{c}=h_{c}^{*}+h_{\text {pile }}^{*},
$$

where $h_{c}^{*}$ is the elastic contact depth, $h_{\text {pile }}^{*}$ is the depth due to the plastic bulk.

Elastic displacements can be calculated according to [15]:

$$
h_{c}^{*}=h_{\max }-\varepsilon \frac{P_{\max }}{S},
$$

for a spherical indenter $\varepsilon=0,75$.

The value of plastic bulk is obtained as a result of finite element analysis:

$$
\frac{h_{\text {pile }}^{*}}{h_{c}^{*}}=0,131 \cdot\left(1-3,243 n+0,079 n^{2}\right)\left(1+6,258 \bar{h}-8,072 \bar{h}^{2}\right)
$$

Earlier in [25], it was also suggested that elastic deformations and plastic "bulk" should be described by separate equations or functions, but this was not realized.

Based on the above data and taking into account the work [6] devoted to the interaction of a rigid sphere with an elastoplastic half-space, the following conceptual model for achieving this goal is proposed. The elastic-plastic contact occurs at $P>P_{y}$, where $P_{y}$ is the critical load at which plastic deformation begins. The degree of loading is characterized by the value $K=P / P_{y}$. In case of elastoplastic deformation, an elastic sinking of the material and the formation of plastic bulk always occur simultaneously. In the loaded state, the profile equation around the contact area is described by the equation $u_{z}(r)$, in the unloaded state, $u_{p}(r)$. Depending on the degree of loading $K$, the surface outside the 
contact area can either be below the original surface (left side of Fig. 1) or above the original surface (right side of Fig. 1), so respectively, the value of the parameter $c^{2}=h_{c} / h$ will be less or more than 1 . When unloaded, the surface outside the contact area will always be higher than the original surface. The depth of the residual crater from the initial surface is $h_{f}=h-w_{0}$, where $w_{0}$ is the elastic restoration of the center of the crater. The height of the pile in the unloaded state is $u_{p}=h_{c}+w_{c}-h$, where $w_{c}$ is the elastic restoration of the contour of the crater (indentation).

\section{Description of the elastic-plastic implantation of the sphere}

To describe the indentation of the sphere, we use the kinetic indentation diagram (Fig. 2). The loading curve is described by the expression

$$
P=C_{l} \bar{h}^{\alpha}
$$

and the unloading curve

$$
P_{e}=C_{u n l}\left(\bar{h}_{e}-\bar{h}_{f}\right)^{\gamma}
$$

where $C_{l}=P_{m} \bar{h}_{m}^{-\alpha}, C_{\text {unl }}=P_{m}\left(\bar{h}_{m}-\bar{h}_{f}\right)^{-\gamma}, \bar{h}_{m}=h_{m} / R, R$-sphere radius; $\alpha=1 \ldots 1.5$, $\gamma=1.3 \ldots 1.5-$ exponents

Differentiating expression (13) from expression (6), we obtain an expression describing the process of elastoplastic penetration of a sphere [5]

$$
\sqrt{h_{c}^{*}}\left(h-h_{f}\right)=\frac{\gamma P}{2 \sqrt{2 R} E^{*}},
$$

where $\gamma=w_{0} / w \approx 1,5$

According to [15],

$$
h_{c}^{*}=h-\frac{\varepsilon P}{S}=h-\frac{\varepsilon}{\gamma}\left(h-h_{f}\right)=h_{f}\left(\frac{h}{h_{f}}\left(1-\frac{\varepsilon}{\gamma}\right)+\frac{\varepsilon}{\gamma}\right)
$$

Substituting expression (15) into (14) and denoting:

$$
\frac{h}{h_{f}}\left(1-\frac{\varepsilon}{\gamma}\right)+\frac{\varepsilon}{\gamma}=y^{2}
$$

we get

$$
y^{3}-y-\frac{P(\gamma-\varepsilon)}{2 E^{*} \sqrt{2 R h_{f}^{1,5}}}=0 .
$$

Using the similarity of deformation characteristics [5], we have 


$$
P=K P_{y} ; \quad h_{f}=\frac{P_{y}(K-1)}{2 \pi R K_{h} \sigma_{y}}
$$

the critical load $P_{y}$ at which plastic deformation begins is determined from the expression:

$$
\frac{P_{y}}{E^{*} R^{2}}=\frac{\pi^{3} K_{y}^{3} \varepsilon_{y}^{3}}{6},
$$

where $K_{y}=p_{0} / \sigma_{y}-$ constant, $p_{0}$ - maximum contact pressure at load $P_{y} ; K_{y}=1.613$

Substituting expressions (18) and (19) in (17), we obtain the equation

$$
y^{3}-y-\frac{6^{0,5} K(\gamma-\varepsilon)}{(K-1)^{1,5}}\left(\frac{K_{h}}{K_{\sigma}}\right)^{1,5}=0,
$$

the free term, which is characterized by dimensionless quantities: the degree of loading $K$ and the constants for a given material $m, \varepsilon, K_{\sigma}$ and the parameter $K_{h}=K_{h}\left(\varepsilon_{y}, n\right)$, which takes into account the characteristics of the hardened material and is determined according to the data [26].

Having the solution $y_{k}$ of equation (20), we find from (16) and (15) the depth of indentation of the sphere and the depth of the elastically contacting part

$$
h=h_{f} \frac{y_{k}^{2}-\varepsilon / \gamma}{1-\varepsilon / \gamma}, \quad h_{c}^{*}=h_{f} \cdot y_{k}^{2}
$$

In view of expressions (18) and (19), we have

$$
\begin{gathered}
\bar{h}_{f}=\frac{h_{f}}{R}=\frac{\pi^{2} K_{y}^{3} \varepsilon_{y}^{2}(K-1)}{12 K_{h}} ; \\
\bar{h}_{c}=\frac{h_{c}}{R}=\frac{\pi^{2} K_{y}^{3} \varepsilon_{y}^{2}(K-1) y_{k}^{2}}{12 K_{h}} \cdot\left(1+0,131 \cdot\left(1-3,243 n+0,079 n^{2}\right)\left(1+6,258 \bar{h}-8,072 \bar{h}^{2}\right)\right) \\
\bar{h}=\frac{h}{R}=\frac{\pi^{2} K_{y}^{3} \varepsilon_{y}^{2}(K-1)}{12 K_{h}} \cdot \frac{y_{k}^{2}-\varepsilon / \gamma}{1-\varepsilon / \gamma} .
\end{gathered}
$$

Thus, all deep-seated characteristics of the indentation of the sphere into the elasticplastic half-space are determined.

\section{Contact geometry description}

Height of unloaded Bulk is

$$
u_{p}=h_{c}-h+w_{c} .
$$

According to [27], taking into account (19) and the fact that $p_{m}=P_{y} K /\left(\pi a^{2}\right)$, 


$$
\bar{w}_{c}=\frac{w_{c}}{R}=\frac{\pi^{2} K_{y}^{3} \varepsilon_{y}^{3} K K_{\beta c}}{6} \cdot\left(2 \bar{h}_{c}-\bar{h}_{c}^{2}\right)^{-\frac{1}{2}}
$$

where $K_{\beta c}=(1+\beta) 2^{2 \beta+1} \mathrm{~B}(\beta+1, \beta+1) \cdot \mathrm{B}(0,5, \beta+1), \mathrm{B}(a, b)-$ beta function

If the indentation of a sphere can be described by expression (12), then the parameter $\beta=\alpha-1[5]$.

The radius of the sphere of the restored crater.

$$
\bar{R}_{c r}=\frac{R_{c r}}{R}=\frac{\bar{h}_{c r}^{2}+2 \bar{h}_{c}-\bar{h}_{c}^{2}}{2 \bar{h}_{c r}}
$$

where $\bar{h}_{c r}$ is the relative depth of the recovered crater,

$$
\begin{gathered}
\bar{h}_{c r}=\bar{h}_{c}-\bar{w}_{0}+\bar{w}_{c}, \\
\bar{w}_{0}=\frac{w_{0}}{R}=\frac{\pi K_{y}^{3} \varepsilon_{y}^{3} K K_{\beta o}}{6} \cdot\left(2 \bar{h}-\bar{h}^{2}\right)^{-\frac{1}{2}}, \\
K_{\beta o}=(1+\beta) 2^{2 \beta+1} \mathrm{~B}(\beta+1, \beta+1) .
\end{gathered}
$$

Based on the analysis of the profiles of the unloaded crateres presented in [21, 28, 29, 30], we make the assumption: the profile of the bulk of the unloaded hole for $r>a$ is described by the expression (Fig. 3)

$$
u_{p}(r)=u_{p}(a)\left(\frac{a_{p}-r}{a_{p}-a}\right)^{2}, \quad a \leq r \leq a_{p} \text {, or } u_{p}(\bar{r})=u(1)\left(\frac{\bar{a}_{p}-\bar{r}}{\bar{a}_{p}-1}\right)^{2}, \quad 1 \leq \bar{r} \leq \bar{a}_{p},
$$

where $\bar{r}=r / a, \quad \bar{a}_{p}=a_{p} / a$.

The profile equation of the unloaded hole (Fig. 3): $z(r)=-R_{c r}+h_{f}+\sqrt{R_{c r}^{2}-r^{2}}$

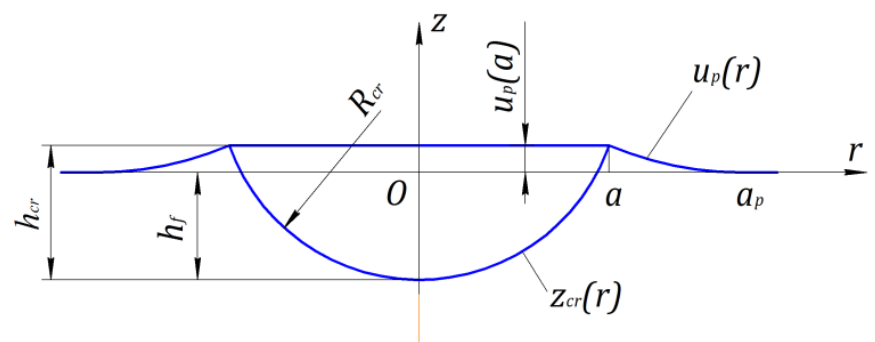

Fig. 3. The surface profiles of the unloaded crater

The value of $a_{p}$ is found from the condition:

$$
\int_{0}^{a} z(r) \cdot r d r+\int_{a}^{a_{p}} u_{p}(r) r d r=0
$$




$$
\bar{a}_{p}=-1+\sqrt{1-\frac{6\left(\bar{R}_{c r}-\bar{h}_{f}\right)}{\bar{u}_{p}(1)}-\frac{4\left[\left(\bar{R}_{c r}^{2}-\left(2 \bar{h}_{c}-\bar{h}_{c}^{2}\right)\right)^{\frac{3}{2}}-\bar{R}_{c r}^{3}\right]}{\bar{u}_{p}(1) \cdot\left(2 \bar{h}_{c}-\bar{h}_{c}^{2}\right)}} .
$$

The elastic displacement $u_{c}(\bar{r})$ of the surface points outside the contact area is described by the expression [27]

$$
\frac{u_{e}(\bar{r})}{R}=\frac{\pi K_{\sigma}^{3} \varepsilon_{y}^{3} K 2^{2 \beta+1}(\beta+1)}{6 \bar{r} \sqrt{2 \bar{h}-\bar{h}^{2}}} \cdot \mathrm{B}(\beta+1, \beta+1) \times \mathrm{B}\left(\frac{1}{2}, \beta+\frac{3}{2}\right){ }_{2} F_{1}\left(\frac{1}{2}, \frac{1}{2} ; \beta+2, \bar{r}^{-2}\right) .
$$

Then the profile of the noncontacting surface in the loaded state is described by the equation

$$
u_{z}(\bar{r})=u_{p}(\bar{r})+u_{e}(\bar{r})
$$

In order to verify the obtained expressions, we considered the profiles for different indentation values $\bar{h}_{r}=h / R$ (Fig. 4b) obtained in [21] as a result of finite element modeling. Similar profiles were obtained (Fig. 4a) using expressions (31), (32) and (34). The values of the coefficient $K_{h}\left(\varepsilon_{y}, n\right)$ were calculated according to the procedure [26] using the values of $h_{r i}$ and $P_{i}$ from [31].

a)

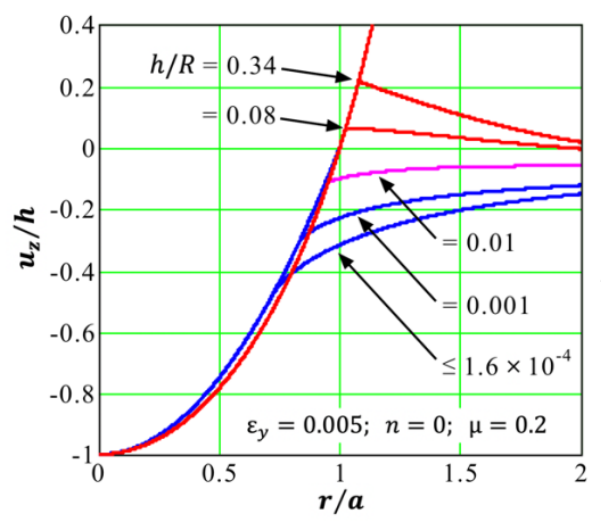

б)

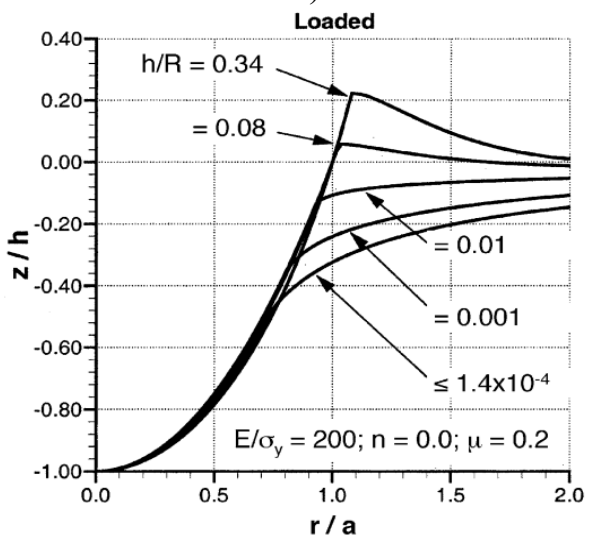

Fig. 4. Half-space profiles for different values of the indentation of the sphere: a) - by expression (44); b) - according to [21]

As follows from fig. 4, there is a fairly good qualitative and quantitative agreement of the results with the data of finite element modeling.

\section{Conclusion}

1. The indentation of a sphere for the entire range of elastic-plasticity is described by a single expression in a dimensionless form depending on the degree of loading $K$, taking into account the characteristics of the hardened material.

2. The authors hypothesis was confirmed [24, 25] that "sink-in / hile-up" effects are separate processes and should be described by separate equations. 
3. A comparison of the obtained results indicates the acceptability of the obtained expressions for engineering calculations of half-space profiles when introducing spherical asperity and the reliability of the assumption made on the bulk profile.

4. The results obtained allow us to determine the amount of clearance per one asperity

$$
V_{i}=2 \pi \int_{a}^{a_{c}}\left(z_{i}(r)-u_{z}(r)\right) r d r,
$$

where $a_{c}=\left(A_{c} / \pi\right)^{0,5}, A_{c}$ is the area per single asperity; $z_{i}(r)$ is the equation describing the $i$-th asperity.

Given the distribution function of spherical asperities along the height of the rough layer, it is possible to determine the density of the gaps when a rigid rough surface is embedded in an elastic-plastic half-space, depending on the applied load and the properties of the half-space material.

5. A similar approach can be used to describe the flattening of a spherical asperity.

\section{References}

1. N.B. Demkin, Contact of rough surfaces (1970)

2. P. Ogar, S. Belokobylsky, D. Gorokhov, Contact and Fracture Mechanics (2018)

3. J.A. Greenwood, J.B.R. Williamson, Proc. Roy.Soc. A295, 301 (1966)

4. I.V.Kragel'skij, N.M. Dobychin, V.S. Kombalov, Basics of calculation the friction and wear (1974)

5. H. Gheadnia, S. A. Pope, R. L. Jackson, D. B. Marghitu, Tribiology International, 93, Part A, 78 (2016)

6. P.M. Ogar, V.A. Tarasov, Adv. Mat. Res., 664, 625 (2013)

7. R. L. Jackson, L. Kogut, Journal of tribology, 128, 209 (2006)

8. H. Ghaednia, X. Wang, S. Saha, Y. Xu, A. Sharma, R. L. Jackson, ASME. Appl. Mech. Rev., 69(6): 060804 (2017)

9. P.M. Ogar, D.B. Gorokhov, A.S. Kozhevnikov, Proc. 2nd Int. Conf. on Modelling, Identification and Control (MIC 2015)

10. P. Ogar, D. Gorokhov, L. Mamaev, V. Fedorov, Adv. Eng. Res, 158, 313 (2018)

11. E. Meyer,. Zeitschrift des Vereins Deutschen Ingenieure, 52, 645 (1908)

12. A. Norbury, T. Samuel, J. Iron Steel Inst., 117, 673 (1928)

13. J. R. Matthews, Acta Materialia, 28, 311 (1980)

14. R. Hill, B. Storakers, A. B. Zdunek, Proc. Royal Soc., A 423, 301 (1989)

15. W. C. Oliver, G. M. Pharr, J. Mat. Res.,. 7 (6), 1564 (1992)

16. W.C. Oliver, G.M. Pharr, J. Mat. Res., 19 (1), 3 (2004)

17. S.I. Bulychev, V.P. Alekhin, M.Kh. Shorshorov. A.P. Ternovskii, Strength Mater., 8 (9), 1084 (1976)

18. B. Taljat, T. Zacharias, T. Kosel, Int. J. Solids and Struct., 35, 4411 (1998)

19. J. Alcala, A. C. Barone, M. Anglada, Acta Materalia,. 48, 3451 (2000)

20. S. Kucharski, Z. Mroz, Mat. Sci. Eng., A.318, 65 (2001)

21. B. Taljat, G. M. Pharr, Int. J. Solids and Struct., 41, 3891 (2004)

22. H. Lee, J.H. Lee, G.M. Pharr, J. Mech. Phys. Solids, 53, 2037 (2005)

23. X. Hernot, O. Bartier, Y. Bekouche, G. Mauvoisin, R. El Abdi, Int. J. Solids and Struct., 43, 4136 (2006)

24. S.H. Kim, B.W. Lee., Y. Choi, D. Kwon, Mat. Sci. Eng., A 415, 59 (2006) 
25. J.H. Ahn, D. Kwon, Mater. J. Res., 16 (11), 3170 (2001)

26. P.M. Ogar, V.A. Tarasov, A.V. Turchenko, Syst. Meth. Technol., 4(12), 29 (2011)

27. P.M. Ogar, V.A. Tarasov, Syst. Meth. Technol., 1, 14 (2010)

28. K. Ai, L.H. Dai, Physics Mech. and Astr., 51 (4), 379 (2008)

29. G.L. Cipriano, Determinacao do coeficiente de encruamento de metais atraves da morfologia das impressoes de dureza na escala macrcopica: diss. PhD (2008)

30. B.D. Monelli, Mechanical Characterization of Metallic Materials by Instrumented Spherical Indentation Testing: diss. PhD (2010)

31. M. Collin, G. Mauvoisin, P. Pilvin, Materials and Desing, 31, 636 (2010) 